Research report

\title{
Synchrony of gaze and stepping patterns in people with Parkinson's disease
}

\author{
Rodrigo Vitório $^{\mathrm{a}, \mathrm{b}, *}$, Lilian T.B. Gobbi ${ }^{\mathrm{b}}$, Ellen Lirani-Silva ${ }^{\mathrm{b}}$, Renato Moraes $^{\mathrm{c}}$, \\ Quincy J. Almeida ${ }^{a}$ \\ a Sun Life Financial Movement Disorders Research E' Rehabilitation Centre, Wilfrid Laurier University, Waterloo, Ontario N2L 3C5, Canada \\ b Posture and Gait Studies Lab, UNESP - Univ Estadual Paulista (Sao Paulo State University), Rio Claro, Sao Paulo State 13506-900, Brazil \\ ' School of Physical Education and Sport at Ribeirao Preto, University of Sao Paulo, Ribeirao Preto, Sao Paulo State 14040-907, Brazil
}

\section{H I G H L I G H T S}

- We studied the synchronism of gaze and stepping patterns in Parkinson's disease.

- Participants walked through paths with foot placement targets (one vs. two targets).

- Patients and healthy participants prioritized the planning of future actions.

- Only patients increased A-P foot placement error in the Two Target condition.

- Patients are dependent on on-line visual information to guarantee an accurate step.

\section{A R T I C L E I N F O}

\section{Article history:}

Received 5 October 2015

Received in revised form 9 March 2016

Accepted 5 April 2016

Available online 6 April 2016

\section{Keywords:}

Gait

Eye tracking

Parkinson's disease

Gaze

\begin{abstract}
A B S T R A C T
The aim of the present study was to test the hypothesis that people with Parkinson's disease (PD) are more dependent than healthy individuals on visual information in an on-line manner to guarantee accurate foot placement into an intended stepping target. Patients with PD and age-matched healthy participants walked along a pathway and were required to step onto either one or two targets during the walk trial. Outcome measures included absolute error (accuracy) and error variability (precision) of foot placement onto the first target, and the time interval between the gaze transfer away from the first target and heel contact on the same target. When there was a single target in the travel path, both groups fixated the target until after heel contact on the target. However, when challenged with an additional target, both groups transferred their gaze from the first target prior to heel contact. Interestingly, only people with PD increased anterior-posterior absolute error (first target) when there was more than one target in the travel path. Premature gaze transfer was associated with decline in stepping accuracy (anteriorposterior absolute error) in people with PD. These findings suggest that both people with PD and healthy individuals prioritize the planning of future actions over the execution of ongoing steps, while walking. Additionally, current findings support the notion people with PD are more dependent on visual feedback to make on-line corrections and adjustments to their foot trajectory in order to guarantee accurate foot placement into an intended stepping target.
\end{abstract}

(C) 2016 Elsevier B.V. All rights reserved.
Abbreviations: PD, Parkinson's disease; MMSE, Mini Mental State Examination; UPDRS, Unified Parkinson's Disease Rating Scale; IRED, infrared light-emitting diode; ANOVA, analysis of variance.

* Corresponding author at: Laboratório de Estudos da Postura e da Locomoção (Posture and Gait Studies Lab), Depto de Educação Física, Universidade Estadual Paulista (UNESP), 24-A Avenue, 1515, Rio Claro, Sao Paulo State 13506-900, Brazil.

E-mail address: vitoriorodrigo@gmail.com (R. Vitório).

\section{Introduction}

It is well documented that people with Parkinson's disease (PD) fall more frequently than healthy individuals [1,2]. Most falls occur during gait in PD and therefore previous research has focused on identifying disease-related deficits in locomotor performance that lead to falls. Several studies have reported foot clearance difficulties during obstacle crossing by people with PD [3-6]. Not surprisingly, tripping over obstacles has been identified as one of the major causes of falls in PD [7]. Since walking over obstacles requires 
visually guided foot placement on specific locations, deficits in sampling and/or processing visual information from the environment might be an important cause of tripping. Importantly, people with PD report basic visual deficits such as impaired visuo-perceptual function and reduced contrast sensitivity $[8,9]$. In addition, eyetracking studies have demonstrated visual sampling impairment in people with PD, such as hypometric voluntary sampling and variable reflexive saccades [10-12]. Most of these previous studies involve sitting in front of a computer, and hence fail to take into consideration how visual impairments may be compounded by movement itself. Then, it is important to investigate diseaserelated visual sampling deficits during walking in people with PD [13].

Walking safely through cluttered environments requires visual identification of obstacles and safe places to step. It is well established that there are strong spatiotemporal relationships between eye and stepping movements during precision walking tasks. For example, gaze stabilization with respect to footfall targets plays a crucial role in the control of visually guided stepping (e.g. obstacle avoidance or foot placement on targets) $[14,15]$. Previously, Chapman and Hollands [14] demonstrated that, when there was more than one stepping constraint in the travel path, in contrast to young and low-risk older adults, older adults categorized as having a high-risk of falling transferred gaze away from the first target prior to completing the step on that same target, in order to fixate on an upcoming target. This early transfer of gaze by high-risk older adults was accompanied by an increase in foot placement variability in the first target. Authors proposed that older adults who are prone to fall may prioritize the planning of future actions over the accurate execution of ongoing movements, which may contribute to an increased likelihood of falls.

To our knowledge, the synchrony between gaze and stepping patterns during walking in people with PD has only been investigated in one study. A recent study from our group [16] demonstrated that both people with PD and healthy individuals use similar gaze behavior to capture visual information from visual cues while walking on a cued pathway with targets for each step (participants were instructed to touch each line with their heels consecutively as they proceed through the trial). Both groups employed equal distribution of on-line (use of visual information available during ongoing step) and feedforward (use of visual information that was viewed ahead of the currently occurring step; to plan ahead) visual control of gait, but people with PD performed less accurate foot placements on the target cues when they employed the feedforward visual control [16]. It is important to note that the use of feedforward control while stepping on specific targets requires relatively intact proprioception (to guide the concurrent steps without visual information of the foot relative to the target, as vision is directed ahead) and storage capacity in the working memory (to remember target position) to guarantee accurate foot placements. The results of our previous study [16] suggest that people with PD may be more dependent on the availability of visual information in an on-line mode to fine tune the accuracy of foot placement on targets; probably to compensate the proprioceptive and working memory deficits usually observed in people with PD [17-19]. However, stepping accuracy was not stressed in the instruction offered to participants in our previous study [16], making it difficult to solidly support this conclusion. As such, it is necessary to study gaze and locomotor behavior of people with PD while performing a task which requires accurate foot placements on desired locations.

In the current study, people with PD and healthy individuals were required to walk through two different travel paths with foot placement targets (one vs. two targets) while wearing a head mounted gaze tracker unit. By manipulating foot placement pattern (travel path with foot placement targets) and monitoring gaze and stepping behavior, it was possible to determine how people with PD and healthy individuals synchronized gaze and stepping patterns during the target-directed gait. The aim of the present study was to test the hypothesis that people with PD are more dependent than healthy individuals on visual information in an on-line manner to guarantee accurate foot placement into an intended stepping target. Since people with PD fall frequently and they demonstrate proprioceptive and working memory deficits, we expected to observe increased foot placement error when patients with PD use feedforward control (early gaze deviation from the target to be stepped).

\section{Material and methods}

\subsection{Participants}

This study adhered to the guidelines of the Declaration of Helsinki, and it was approved by the Research Ethics Board at Wilfrid Laurier University. All participants signed a consent form before participating in the experimental protocol. Twenty four patients with PD (12 males) and 18 age-matched control subjects ( 8 males) were recruited from a database at the Sun Life Financial Movement Disorders Research and Rehabilitation Centre at Wilfrid Laurier University in Waterloo, Ontario, Canada. Patients were selected on the criteria of having confirmed PD diagnosis from at least one neurologist. A movement disorder specialist performed a clinical assessment in order to test patients on the Unified Parkinson's Disease Rating Scale (UPDRS, [20]), the Hoehn and Yahr Rating Scale (H\&Y, [21]) and the Mini Mental State Examination (MMSE, [22]). Patients were tested in the ON state of medication (approximately $1 \mathrm{~h}$ after having taken a dose). The levodopa equivalent daily dose was calculated according to Tomlinson's suggestions [23]. Patients with PD who experienced freezing of gait were excluded from the study. Participants in both groups had no other neurological, musculoskeletal or cardiorespiratory disturbances that could impair walking ability. All participants were cognitively preserved (scored at least 27 on the MMSE).

\subsection{Protocol and data analysis}

Participants were required to walk along a pathway $8 \mathrm{~m}$ long, at a self-selected pace, and step on one of two target conditions (Fig. 1). The "One target" condition required participants to place their right foot onto target 1 . The "Two target" condition required participants to place their right foot onto target 1 and then subsequently their left foot onto target 2. Participants were instructed to place each foot accurately in the central area of the targets. Five trials in each condition per participant (10 trials) were performed in a random order. The targets were rectangular and made of lightweight foam rubber measuring $19 \mathrm{~cm} \times 41.5 \mathrm{~cm}$ [14]. The targets were positioned at the central area of the pathway at constant distance apart (A-P separation $=180 \mathrm{~cm}, \mathrm{M}-\mathrm{L}$ separation $=45 \mathrm{~cm}$ ). Prior to the $\mathrm{start}$ of each trial, participants were instructed to close their eyes. Each trial began on a verbal signal "Ready? Go!", at which point participants were instructed to open their eyes and start the experimental task.

Foot 3-D kinematic data were collected using an Optotrak wireless system (Northern Digital Inc., Waterloo, Ontario, Canada) with five position sensor cameras (one placed on the wall at the end of the pathway, two placed on the right and two placed on left side of the pathway). A total of six infrared light-emitting diodes (IREDs) were attached bilaterally to the following anatomic landmarks: (a) first metatarsal and fifth metatarsal heads and (b) back of the calcaneous. These IREDs were tracked at a sampling frequency of $120 \mathrm{~Hz}$. The following gait variables were calculated for the step ending in 


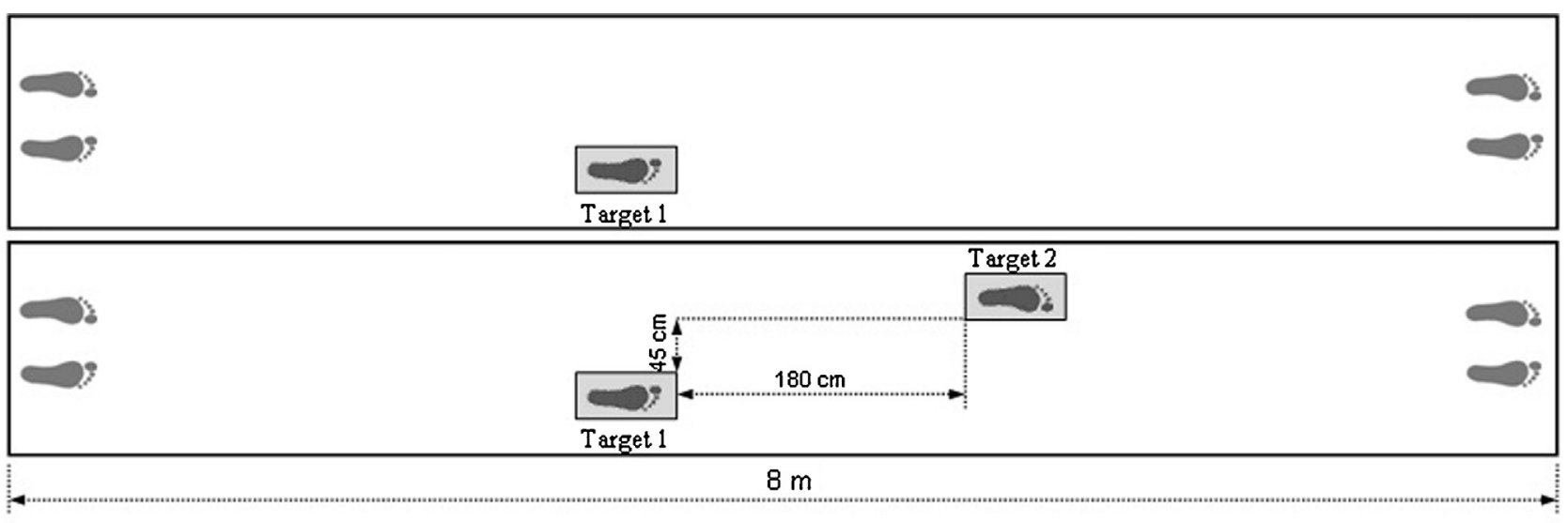

Legend: One Target condition (top); Two Target condition (bottom).

Fig. 1. Schematic diagram of experimental task.

heel contact at target 1: absolute error $(\mathrm{cm})$ : defined as the distance between the centre of the foot from the centre of target 1 (separate measures in M-L and A-P planes); error variability $(\mathrm{cm})$ : standard deviations of absolute error in target 1 .

Gaze behavior was recorded by using a high speed head mounted eye tracker unit (ASL H6, ASL, Bedford, MA, USA) in all conditions at $120 \mathrm{~Hz}$. The eye tracker system was calibrated using the 9-point calibration method. Participants fixated their gaze on 9 points displayed in a 3 by 3 grid. Calibration was also checked periodically between trials. Gaze data was temporally synchronized with kinematic data by an electronic device which started data recording at the same time in both kinematic and gaze tracking systems. The time interval between the gaze transfer away from target 1 (defined as the first frame in which gaze was located out of the target 1) and heel contact on target 1 was calculated. Positive values indicate gaze transfer away from target 1 prior to heel contact and negative values indicate gaze transfer away from target after the heel contact.

For demographic data, unrelated sample student t-tests were employed for between-group comparisons. Two-way ANOVAs (group $\mathrm{x}$ condition) were carried out, with repeated measures in the condition factor. Significant interactions were followed up with post-hoc comparisons using Tukey's HSD procedure. Correlation analyses were conducted to assess the relationship between clinical data (for people with PD), gaze behavior and stepping performance. All statistical analyses were run by the software SPSS for Windows 15.0. $P$-value was set to 0.05 .

\section{Results}

The two groups were not significantly different in MMSE or any demographic variables (Table 1). Clinical data for the individuals with PD are also reported in Table 1, and they had mild to moderate disease severity measured by the UPDRS motor section. Due to problems with data collection using the eye tracker unit, statistical analyses of gaze data included data of 21 patients with PD and 16 healthy individuals.

A significant interaction between group and target condition was observed for anterior-posterior absolute error $\left[F_{(1,40)}=4.118\right.$, $p=0.049$ ] (Fig. 2A). Post hoc tests revealed that the two groups had similar anterior-posterior absolute error for the One Target condition $(p=0.693)$, but only people with PD increased the anterior-posterior absolute error in the Two Target condition $(p=0.001$; Fig. 2A). No other group main effect or interactions between factors were observed for other dependent variables.
A main effect of condition was observed for medio-lateral absolute error $\left[F_{(1,40)}=11.413, p=0.002\right]$, medio-lateral error variability $\left[\mathrm{F}_{(1,40)}=4.665, p=0.037\right]$, and time interval between the gaze transfer away from target 1 and heel contact on target $1\left[F_{(1,35)}=9.314\right.$, $p=0.004]$. Both people with PD and healthy individuals showed larger medio-lateral absolute error and medio-lateral error variability in the Two Target than in the One Target condition (Fig. 2). Additionally, participants transferred their gaze away from target 1 prior to heel contact during the One Target condition and after heel contact during the Two Target condition (Fig. 3).

Correlation analyses run for people with PD failed to identify significant relationships between clinical data and stepping performance or gaze behavior. A trend for significant relationship was observed between H\&Y stage and anterior-posterior absolute error $(\mathrm{r}=0.402, p=0.07)$. There was a modest but significant correlation between the interval between gaze transfer away from target 1 and heel contact on target 1 and medio-lateral absolute error for both people with $\operatorname{PD}(\mathrm{r}=0.283, p=0.003)$ and healthy participants $(r=0.383, p<0.001)$. Furthermore, there was a modest but significant correlation between the interval between gaze transfer away from target 1 and heel contact on target 1 and anterior-posterior absolute error for people with PD $(r=0.236, p=0.015)$.

\section{Discussion}

The aim of the present study was to test the hypothesis that people with PD are more dependent than healthy individuals on visual information in an on-line manner to guarantee accurate foot placement into an intended stepping target. Healthy individuals completed the task with minimal anterior-posterior absolute error irrespective of the task complexity. In contrast, patients with PD showed a significant increase in anterior-posterior absolute error when there were multiple stepping targets in the travel path. With regarding to medio-lateral direction, both groups increased mediolateral absolute error and variability in the Two Target condition. These findings suggest that the complexity of required walking conditions affects medio-lateral accuracy and precision in both people with PD and healthy individuals. In opposition, the anteriorposterior accuracy of foot placement on target was affected by the multiple step constraint only in patients with PD. In agreement, Chapman and Holland $[14,15]$ demonstrated that when there was more than one stepping constraint in the travel path, in contrast to young and low-risk older adults, older adults categorized as having a high-risk of falling increased anterior-posterior foot placement variability. It seems that deficits in accuracy and precision of foot placement in the anterior-posterior direction are more 
Table 1

Characteristics of Parkinson's disease patients and control group.

\begin{tabular}{|c|c|c|c|}
\hline Demographic variable & PD & Controls & Student $\mathrm{t}$ test \\
\hline age (years) & $65.26 \pm 7.76$ & $64.17 \pm 8.38$ & $\mathrm{t}_{40}=-0.437, p=0.664$ \\
\hline body mass (kg) & $78.29 \pm 14.67$ & $76.33 \pm 14.35$ & $\mathrm{t}_{40}=-0.432, p=0.668$ \\
\hline body height $(\mathrm{cm})$ & $170.89 \pm 8.72$ & $169.66 \pm 8.87$ & $\mathrm{t}_{40}=-0.446, p=0.658$ \\
\hline MMSE (score) & $29.67 \pm 1.05$ & $29.56 \pm 0.86$ & $\mathrm{t}_{40}=-0.367, p=0.716$ \\
\hline UPDRS-III (score) & $18.96 \pm 7.45$ & & \\
\hline Hoehn and Yahr stage & $1.79 \pm 0.62$ & & \\
\hline levodopa equivalent daily dose (mg) & $909.79 \pm 487.72$ & & \\
\hline years since diagnosis & $9.76 \pm 3.97$ & & \\
\hline
\end{tabular}

A

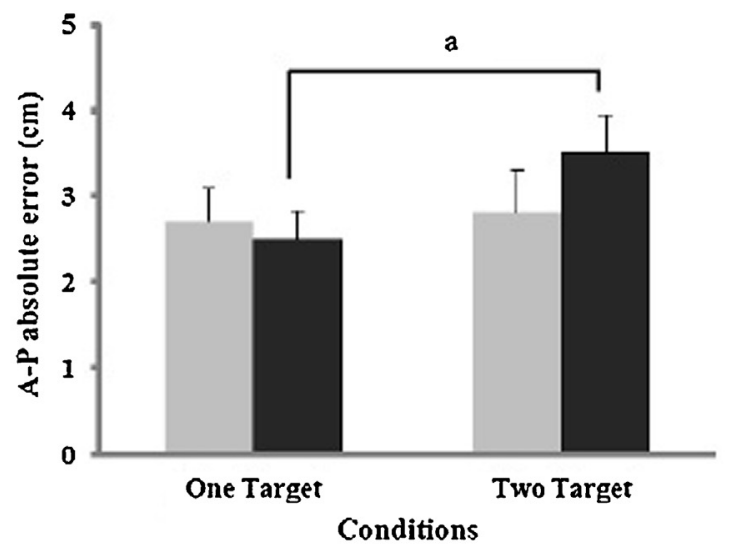

C

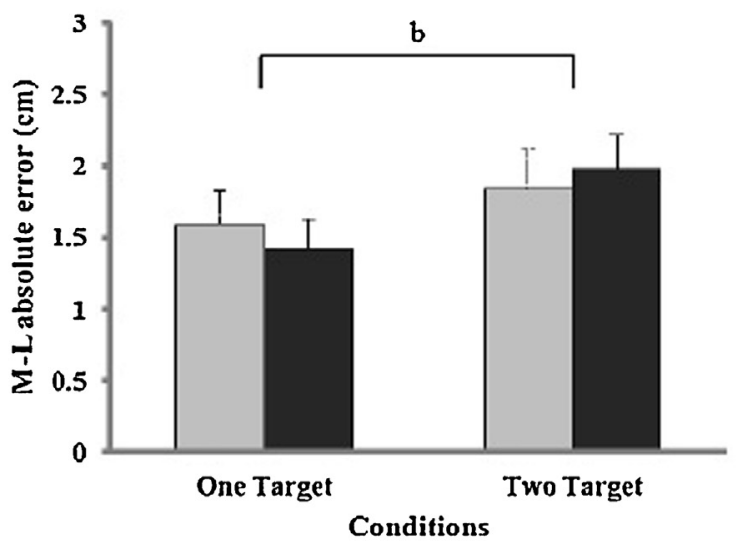

$\mathbf{B}$

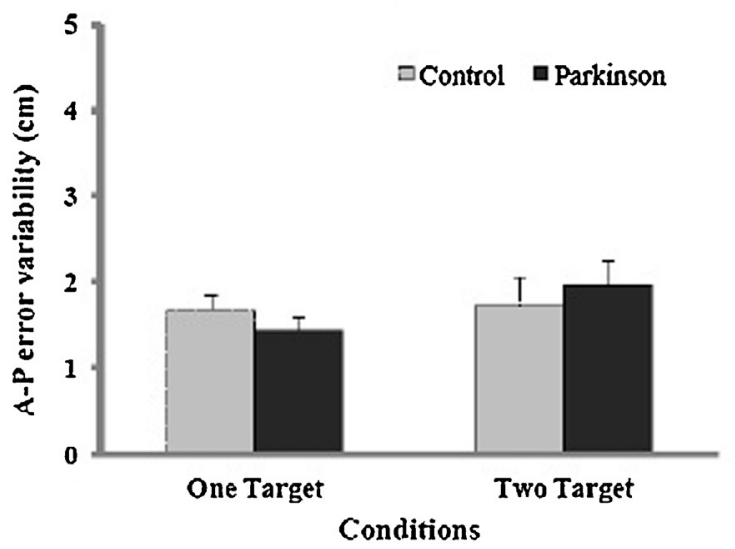

D

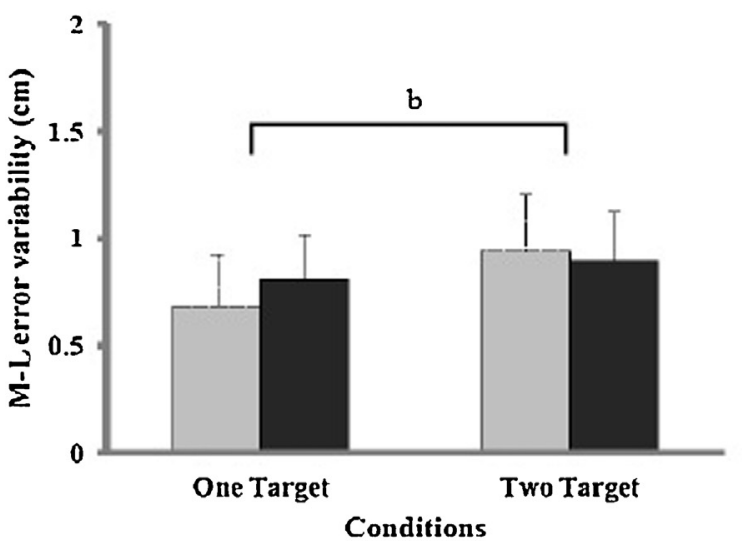

Legend: Anterior-posterior absolute error (A) and error variability (B). Medio-lateral absolute error $(C)$ and error variability (D). a: indicates significant difference between One Target and Two Target conditions for patients with PD; $b$ : indicates significant difference between One Target and Two Target conditions for both groups.

Fig. 2. Means and standard errors of absolute error and error variability for each group on each experimental condition.

dangerous than in the medio-lateral direction. The results of the current study suggest that the decrease in anterior-posterior foot placement accuracy observed in patients with PD may be partially responsible for the increased incidence of trips and falls in this population during walking in travel paths with more than one stepping constraint. This explanation is consistent with previous findings from obstacle avoidance tasks [3,5], which have demonstrated that people with PD have difficulty lengthening their step over the obstacle (anterior-posterior direction) rather than increasing foot height (vertical direction).

Interestingly, there were no between group difference or interaction between group and target condition for the time interval between the gaze transfer away from target 1 and heel contact on target 1 . The main effect of condition revealed that both groups 


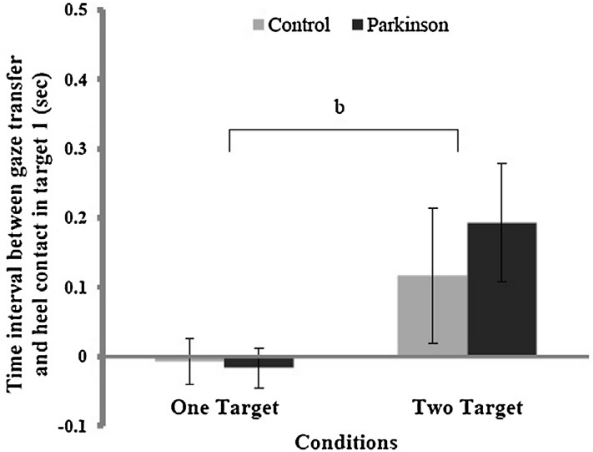

Legend: $b$ : indicates significant difference between One Target and Two Target condition for both groups.

Fig. 3. Means and standard errors of the time interval between gaze transfer and heel contact in target 1 for each group on each experimental condition.

transferred their gaze away from target 1 after heel contact $(11 \mathrm{~ms})$ during the One Target condition and prior to heel contact (155 ms) during the Two Target condition. These findings suggest that the complexity of required walking conditions affected the synchronization of gaze and stepping patterns in the same way in both groups, with an early gaze deviation from target 1 when there was more than one stepping constraint in the travel path. It might be the case that participants perceived the upcoming stepping constraint as a threat to their balance, suggesting that anxiety potentially mediated gaze behavior in the Two Target condition, although anxiety was not measure in the current study. Anxiety has been shown to be a significant predictor of decline in successful obstacle avoidance during locomotion in older adults [24]. Anxiety also adversely affects gaze control, reducing gaze stability on visual targets [25]. Another possible explanation for the early transfer of gaze exhibited by the participants in the Two Target condition relates to the time needed to program changes to the ongoing stepping pattern. Older adults may look away before completing the ongoing step to allow sufficient time to plan subsequent stepping movements. Current findings are partly consistent with findings from Chapman and Hollands [15], who demonstrated that only older adults categorized as high-risk of falling transferred gaze away from the first target prior to completing the step into that target in trials with multiple step constraints in the path. Although it did not reach significance, it is important to note that patients with PD transferred their gaze away from the first target $76 \mathrm{~ms}$ earlier than healthy individuals during the Two Target condition (PD: $193 \mathrm{~ms}$; Control: $117 \mathrm{~ms}$; Fig. 3). This may have influenced foot placement accuracy observed (impaired anterior-posterior accuracy observed for people with PD). Another possibility is that the control group of current study could not be classified as 'low-risk of falling' as in the studies by Chapman and Hollands [14,15]. For example, we did not record fall history or balance. In the study by Chapman and Hollands [14], low-risk older adults transferred their gaze away from the first target $10 \mathrm{~ms}$ prior to heel contact whereas high-risk older adults transferred their gaze away significantly sooner $(100 \mathrm{~ms})$. Gaze deviation reported for high-risk older adults is similar to gaze deviation observed for our control group $(117 \mathrm{~ms})$. These findings support the idea that control participants of current study did not behavior as 'low-risk of falling' and, as a consequence of early gaze deviation, they increased foot placement error and variability in the medio-lateral direction.

Although both groups transferred gaze away from the first target prior to heel contact into that target in the Two Target condition, only patients with PD increased anterior-posterior absolute error in this condition. The link between these results was confirmed by correlation analysis. Significant correlation was revealed between interval between gaze transfer away from target 1 and heel contact on target 1 and anterior-posterior absolute error for people with PD only (there was significant correlation between gaze timing and medio-lateral absolute error for both people with PD and healthy participants). In other words, there was a significant association between transferring gaze away early from the target 1 and an impairment on foot placement accuracy on both directions for people with PD (compared to medio-lateral direction for healthy participants). Together, these findings support the notion that people with PD are more dependent than healthy individuals on visual feedback to make on-line corrections and adjustments to their foot trajectory, that are likely due to proprioceptive $[17,18]$ and/or working memory deficits [20], that impair the ability of individuals with PD to complete an accurate foot placement to an intended step target. It might be the case that feedforward mechanism of motor control is impaired in people with PD. Feedforward control refers to the use of visual information that was sampled before the execution of current step [26] and requires a relatively intact storage capacity in the working memory, which is impaired in patients with PD and then could lead to a less accurate motor response. If this was the case, exproprioceptive visual information (information of the body and limbs relative to the environment; i.e. watching your feet while deliberately stepping into puddles) would be crucial to guarantee accuracy of foot placement on targets in PD. This might lead us to suggest that rehabilitation programs should train patients with PD to keep looking at the intended stepping location until the moment of heel contact with a target location. Such strategy would minimize foot placement error and avoid tripping on obstacles during activities of real life. To date, Young and Hollands [27] demonstrated that this strategy is effective in older adults: Following intervention, participants delayed gaze transfer from the first target until after heel contact and this change in behavior resulted in a significant reduction in stepping errors.

Future researches are encouraged to investigate in details how cognitive, emotional and proprioceptive impairments affect gaze control during real-world walking activities. PD is associated with impaired motor control and a range of cognitive, psychiatric and sensory deficits. That being said, studying PD as a model may help to further clarify the links between motor, cognitive, emotional and gaze impairments.

\section{Conclusion}

Both people with PD and healthy participants prioritize the planning of future actions over the execution of ongoing step while walking in travel paths with multiple stepping constraints. However, people with PD are more dependent than healthy individuals on visual information in an on-line manner (until the moment of heel contact) to guarantee accurate foot placement into an intended stepping target. Lastly, decrease in anterior-posterior foot placement accuracy may be the cause of some trips and falls in people with PD during walking in cluttered environments.

\section{Acknowledgements}

We would like to thank Patricia Freeman for participant recruitment. We also thanks all participants for their voluntary participation. This research was supported in part by the Sao Paulo Research Foundation (Fundação de Amparo à Pesquisa do Estado de São Paulo) as a Ph.D. scholarship to Rodrigo Vitório (FAPESP \#12/03456-2; \#10/07040-0), as well as the Canada Foundation for Innovation, Connaught Fund, Kitchener \& Waterloo Community Foundation, and Sun Life Financial to Quincy J. Almeida. 


\section{References}

[1] B.H. Wood, J.A. Bilclough, A. Bowron, R.W. Walker, Incidence and prediction of falls in Parkinson's disease: a prospective multidisciplinary study, J. Neurol. Neurosurg. Psychiatry 72 (2002) 721-725.

[2] L.Z. Rubenstein, K.R. Josephson, Falls and their prevention in elderly people: what does the evidence show? Med. Clin. North Am. 90 (2006) 807-824.

[3] B. Galna, A.T. Murphy, M.E. Morris, Obstacle crossing in people with Parkinson's disease: foot clearance and spatiotemporal deficits, Hum. Movement Sci. 29 (2010) 843-852.

[4] E.L. Stegmöller, T.A. Buckley, C. Pitsikouliset, E. Barthelemy, R. Roemmich, C.J. Hass, Postural instability and gait impairment during obstacle crossing in Parkinson's disease, Arch. Phys. Med. Rehabil. 93 (2012) 703-709.

[5] R. Vitório, F. Pieruccini-Faria, F. Stella, S. Gobbi, L.T.B. Gobbi, Effects of obstacle height on obstacle crossing in mild Parkinson's disease, Gait Posture 31 (2010) 143-146.

[6] R. Vitório, E. Lirani-Silva, F.A. Barbieri, V. Raile, F. Stella, L.T.B. Gobbi, Influence of visual feedback sampling on obstacle crossing behavior in people with Parkinson's disease, Gait Posture 38 (2013) 330-334.

[7] H. Stolze, S. Klebe, C. Zechlin, C. Baecker, L. Friege, G. Deuschl, Falls in frequent neurological diseases. Prevalence, risk factors and aetiology, J. Neurol. 251 (2004) 79-84.

[8] S. Davidsdottir, A. Cronin-Golomb, A. Lee, Visual and spatial symptoms in Parkinson's disease, Vision Res. 45 (2005) 1285-1296.

[9] D. Verbaan, J. Marinus, M. Visser, S.M. Van Rooden, A.M. Stiggelbout, H.A. Middelkoop, et al., Cognitive impairment in Parkinson's disease, J. Neurol. Neurosurg. Psychiatry 78 (2007) 1182-1187.

[10] J.M. Chambers, T.J. Prescott, Response times for visually guided saccades in per-sons with Parkinson's disease: a meta-analytic review, Neuropsychologia 48 (2010) 887-899.

[11] S. Van Stockum, M.R. Macaskill, T.J. Anderson, Impairment of voluntary saccades and facilitation of reflexive saccades do not co-occur in Parkinson's disease, J. Clin. Neurosci. 19 (2012) 1119-1124.

[12] T.J. Anderson, M.R. Macaskill, Eye movements in patients with neurodegenerative disorders, Nature Rev. Neurol. 9 (2013) 74-85.

[13] S. Stuart, L. Alcock, B. Galna, S. Lord, L. Rochester, The measurement of visual sampling during real-world activity in Parkinson's disease and healthy controls: a structured literature review, J. Neurosci. Methods 222 (2014) $175-188$.

[14] G.P. Chapman, M.A. Hollands, Evidence for a link between changes to gaze behaviour and risk of falling in older adults during adaptive locomotion, Gait Posture 24 (2006) 288-294

[15] G.P. Chapman, M.A. Hollands, Evidence that older adult fallers prioritise the planning of future stepping actions over the accurate execution of ongoing steps during complex locomotor tasks, Gait Posture 26 (2007) 59-67.
[16] R. Vitório, E. Lirani-Silva, F. Pieruccini-Faria, R. Moraes, L.T.B. Gobbi, Q.J. Almeida, Visual cues and gait improvement in Parkinson's disease: which piece of information is really important? Neuroscience 277 (2014) 273-280.

[17] O.J. Almeida, J.S. Frank, E.A. Roy, M.E. Jenkins, S. Spaulding, A.E. Patla, M.S. Jog, An evaluation of sensorimotor integration during locomotion toward a target in Parkinson's disease, Neuroscience 134 (2005) 283-293.

[18] J. Konczak, D.M. Corcos, F. Horak, H. Poizner, M. Shapiro, P. Tuite, J. Volkmann, M. Maschke, Proprioception and motor control in parkinson's disease, J. Mot. Behav. 41 (2009) 543-552.

[19] E.Y. Lee, N. Cowan, E.K. Vogel, T. Rolan, F. Valle-Inclán, S.A. Hackley, Visual working memory deficits in patients with Parkinson's disease are due to both reduced storage capacity and impaired ability to filter out irrelevant information, Brain 133 (2010) 2677-2689.

[20] C.G. Goetz, B.C. Tilley, S.R. Shaftman, G.T. Stebbins, S. Fahn, P. Martinez-Martin, W. Poewe, C. Sampaio, M.B. Stern, R. Dodel, B. Dubois, R. Holloway, J. Jankovic, J. Kulisevsky, A.E. Lang, A. Lees, S. Leurgans, P.A. LeWitt, D. Nyenhuis, C.W. Olanow, O. Rascol, A. Schrag, J.A. Teresi, J.J. van Hilten, N. LaPelle, Movement disorder society UPDRS revision task force: movement disorder society sponsored revision of the unified parkinson's disease rating scale (MDS-UPDRS): scale presentation and clinimetric testing results, Mov. Disord. 23 (2007) 2129-2170.

[21] M.M. Hoehn, M.D. Yahr, Parkinsonism: onset, progression and mortality, Neurology 17 (1967) 73-581.

[22] M.F. Folstein, S.E. Folstein, P.R. McHugh, "Mini-mental state”. A practical method for grading the cognitive state of patients for the clinician, J. Psychiatr. Res. 12 (1975) 189-198.

[23] C.L. Tomlinson, R. Stowe, S. Patel, C. Rick, R. Gray, C.E. Clarke, Systematic review of levodopa dose equivalency reporting in Parkinson's disease, Mov. Disord. 25 (2010) 2649-2653.

[24] C.C. Persad, B. Giordani, H.C. Chen, J.A. Ashton-Miller, N.B. Alexander, C.S. Wilson, S. Berent, K. Guire, A.B. Schultz, Neuropsychological predictors of complex obstacle avoidance in healthy older adults, J. Gerontol. B. Psychol. Sci. Soc. Sci. 50 (1995) 272-277.

[25] J.P. Staab, The influence of anxiety on ocular motor control and gaze, Curr Opin. Neurol. 27 (2014) 118-124.

[26] A.E. Patla, How is human gait controlled by vision, Ecol Psychol 10 (1998) 287-302.

[27] W.R. Young, M.A. Hollands, Can telling older adults where to look reduce falls: evidence for a causal link between inappropriate visual sampling and suboptimal stepping performance, Exp. Brain Res. 204 (2010) 103-113. 